\title{
Initial experience with a homemade "hybrid-tome" for needle-knife precut in patients with difficult biliary cannulation $\nabla$
}

\section{()ㅇㅇ}

\author{
Authors

\section{Institutions} \\ 1 Digestive Medicine and Endoscopy Division, Academical \\ Hospital “la Zarzuela," Francisco de Vitoria University, \\ Madrid, Spain \\ 2 Digestive Endoscopy Unit, Academical Hospital “la \\ Moncloa," Madrid, Spain
}

Emilio J. De la Morena Madrigal1,2, Isabel Rodríguez García ${ }^{1}$, Ana Belén Galera Ródenas ${ }^{1}$, Elena Pérez Arellano ${ }^{1}$

submitted 4.11 .2020

accepted after revision $\quad 23.6 .2021$

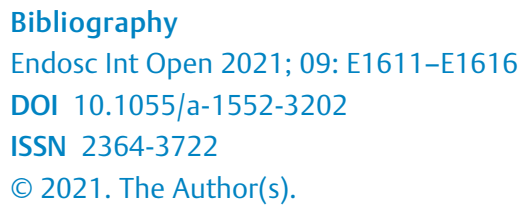
Commons Attribution-NonDerivative-NonCommercial License, permitting copying and reproduction so long as the original work is given appropriate credit. Contents may not be used for commercial purposes, or adapted, remixed, transformed or built upon. (https://creativecommons.org/licenses/by-nc-nd/4.0/)

Georg Thieme Verlag KG, Rüdigerstraße 14,

70469 Stuttgart, Germany

Corresponding author

Emilio J. De la Morena Madrigal., Academical Hospital “la Zarzuela”, C/ pléyades 25, 28023 Aravaca-Madrid, Spain Fax: +0034915858022 emorena@hotmail.es

\section{ABSTRACT}

Background and study aims Current clinical guidelines recommend needle-knife precut (NKP) as the primary and best method for performing a biliary cannulation (BC) when simple techniques fail and the criteria are met for difficult BC (DBC). However, many endoscopists avoid or defer early NKP in favor of alternative, simpler techniques that have not been shown to be either safer or more effective. Our goal is to test a device that provides the needle-knife papillotome (NKPT) with traction capability and which can facilitate the learning and execution of NKP.

Patients and methods This was a descriptive bicentric observational study of a personal cohort of patients undergoing early NKP to analyse the efficacy and safety of the technique with a "hybrid-tome" (HT) built using the isolated core of a NKPT and a conventional canulotome.

Results Over a 4-year period, we performed 43 NKPs with the $\mathrm{HT}$, achieving BC in one or two steps in all cases. The $100 \%$ technical success was matched by a $95 \%$ clinical success rate. We recorded 11 adverse events (23\%): five hemorrhages, four pancreatitis, and two cholangitis. In addition to the objective data, we confirmed that HT facilitates alignment with the duodenal papilla and the execution of pre-cutting, especially if the papilla is intradiverticular or hidden by folds.

Conclusions The HT tested seems to help trained endoscopists to perform NKP, especially in some anatomic situations, which can improve compliance with the guidelines recommended for early NKP in patients with DBC.

\section{Introduction}

Biliary cannulation (BC) is an indispensable technical prerequisite for performing a papillotomy and all transpapillary retrograde biliary procedures. Simple techniques achieve high rates of $\mathrm{BC}$, but never $100 \%$. There is no absolute consensus on the definition of difficult BC (DBC), but regardless, clinical guidelines [1,2] recommend needle-knife precut (NKP) as the primary and best option for achieving $B C$, whether or not unintentional pancreatic cannulation (UPC) has occurred. In the first case, there are technical alternatives, such as the "double guidewire," transpancreatic precutting (TPP), and NKP assisted by a previously implanted pancreatic stent, but none of these takes precedence over simple NKP because there is no evidence of their superiority and, in some cases, the findings actually favor NKP [3].

The key to employing NKP with maximum safety and minimum morbidity (in the form of post-endoscopic retrograde cholangiopancreatography acute pancreatitis [PAP]) lies in it being practiced promptly, at an early stage, following the indi- 
cations established for DBC [4-7]. However, many endoscopists with varying degrees of experience put off performing an NKP in favor of strategies that prolong cannulation time, papillary trauma, and the risk of PAP. The infrequent and delayed use of NKP is accompanied by reduced efficacy and increased morbidity, both of which generate a vicious cycle that is detrimental to patients.

Two closely related factors that explain the limited popularity of NKP among these endoscopists are:

1. The difficulty of the technique, which involves rectifying the position of the duodenoscope and a combination of movements that have to be even more precise than those for a simple cannulation and standard papillotomy.

2. The difficulty of learning the technique and acquiring sufficient competence during the supervised training phase, especially if the trainer is also opposed to the early use of NKP when it is indicated.

Beyond accumulating scientific evidence in favor of the technique, the key to breaking the vicious cycle mentioned above and generating a virtuous one, where the appropriate and timely use of NKP increases the rate of BC and reduces PAP, may lie in making it easier to practice and learn the technique by further developing the needle-knife papillotome (NKPT). Urged years ago by some of the most prestigious endoscopists [8], this call seems not to have been heard by the medical industry.

A hypothetical way to force the evolution of NKPT is to continue along the path that led from simple cannula to the canulotome (CT), shortened form of the term "cannulation papillotome," providing it with traction capability, and therefore, facilitating its alignment with the cut planes and axes and requiring less repositioning of the duodenoscope.

\section{Materials and methods}

The main goal of this study was to demonstrate the technical feasibility of a homemade "hybrid-tome" (HT) handmade from a conventional CT and NKPT. Our secondary objectives were to evaluate its efficacy and safety in appropriate NKP in patients with DBC. To do this, we conducted a retrospective analysis of prospectively recorded data on ERCP performed in two private hospitals in Madrid ( $\mathrm{M}$ and $\mathrm{Z}$ ) over a 4-year period by a single endoscopist (EJMM) with prior experience of more than 2500 ERCPs and 250 NKPs. In hospital M, TJF-160VR duodenoscopes (Olympus, Tokyo, Japan) and HF-120 diathermy generators (Olympus, Tokyo, Japan) were used, while in hospital Z, ED3470TK, 3490TK and 34-i10T2 duodenoscopes (Pentax, Tokyo, Japan) and ICC-200 and VIO-200S generators (ERBE, Tübingen, Germany) were used. When performing the NKP, mixed current was used in "endocut" mode without modifying the parameters set for traction papillotomy.

\section{"Hybrid-tomes"}

The HT was constructed from the insulated metal core of a Huibregtse HPC-2 NKPT (Cook Medical, Bloomington Indiana, United States), which was inserted through the guide channel of a conventional $\mathrm{CT}$ with a Luer connector at the mouth of the

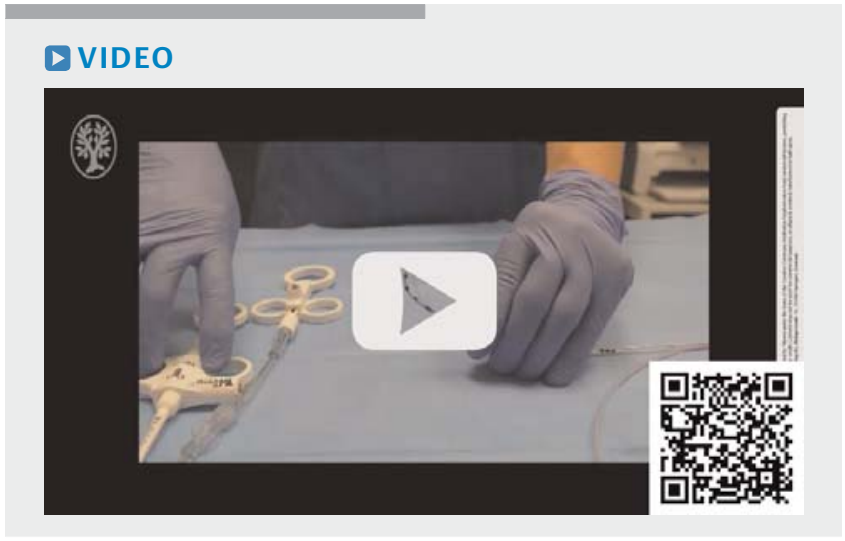

Video 1 Ex vivo performance of the CC-HT.
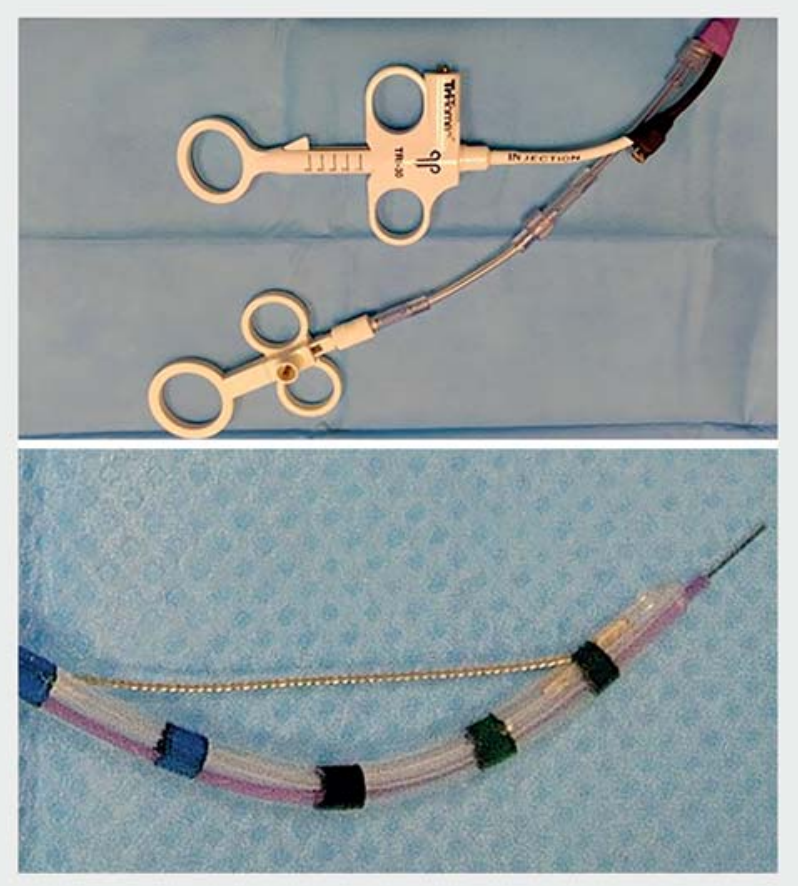

Fig. 1 The CC-HT. Two pieces were combined to adapt the cannulotome guidewire channel to the needle-knife core length.

channel ( $\vee$ Video $\mathbf{1}$ ). Because the lengths of the two devices are not consistent, the channel of the CT was extended by using one or more serial connectors until only the needle emerged from the tip when the handle was in the extended position ( Fig. 1). The CT we used were Clever-Cut 3V (Olympus, Tokyo, Japan) (OC-HT), True-Tome 44 (Boston Scientific, Natick Massachusetts, United States) (BSC-HT) and Tri-Tome 30 (Cook Medical, Bloomington Indiana, United States) (CC-HT) devices ( Fig. 2). 

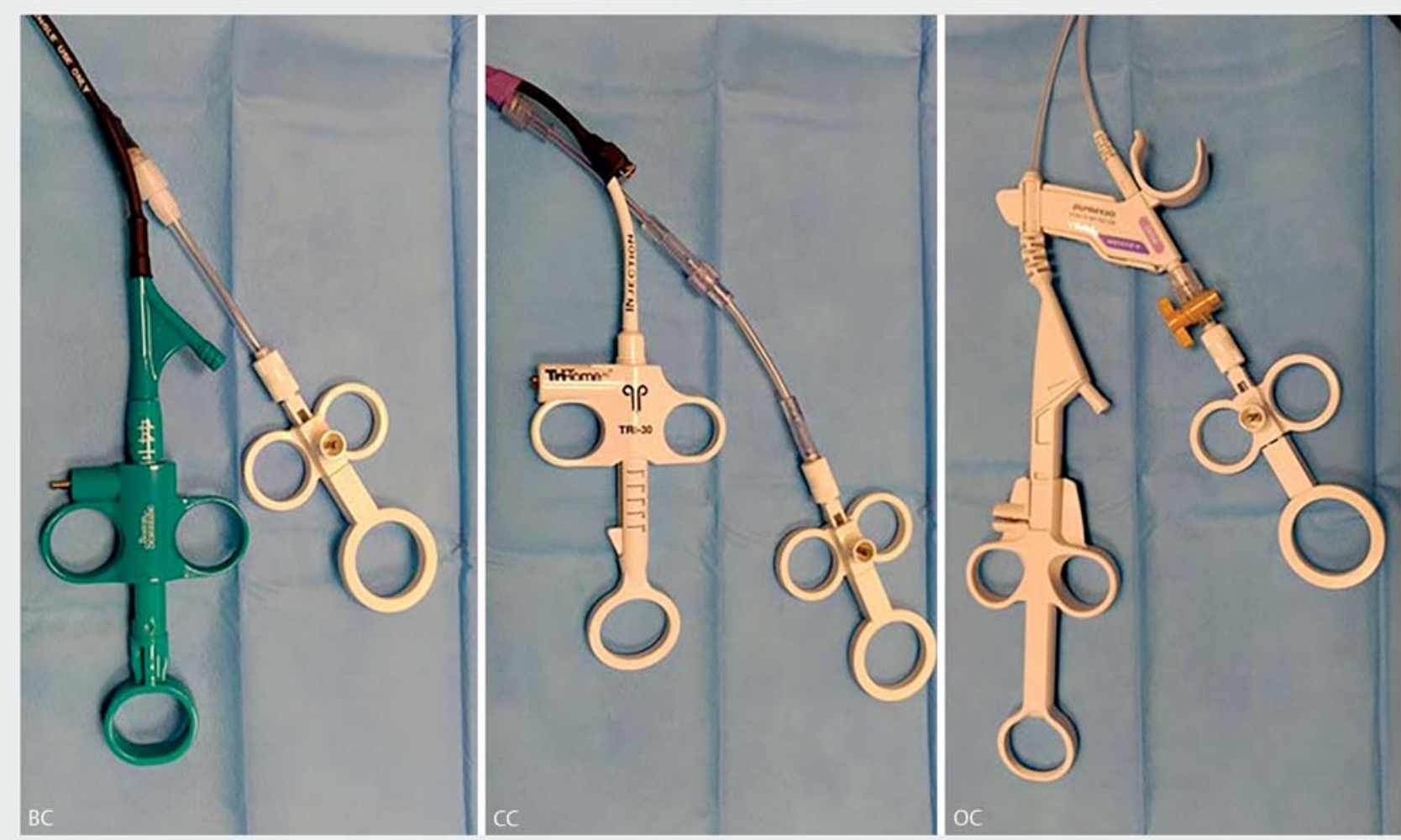

- Fig. 2 The BSC (Boston Scientific-Cook), CC (Cook-Cook) and OC (Olympus-Cook) HT. The different pieces used to adapt either cannulotome guidewire channel to the needle-knife core length are shown.

\section{Difficult biliary cannulation definition and cannulation strategy}

We consider DBC the failure of $B C 10$ minutes after the first contact between the $\mathrm{CT}$ and the papilla or the incidence of three unwanted guidewire pancreatic cannulations. Our BC strategy has been published previously [9]. Briefly, we attempt simple cannulation using $\mathrm{CT}$ and hydrophilic guidance for a maximum of 10 minutes. If we do not achieve BC (or UPC) we perform NKP. If, after the NKP, we do not achieve BC in another 10 minutes, we interrupt the procedure to complete it in a second step (after 3-7 days). If during the attempt at BC, a UPC occurs, we opt to perform a TPP or remove the guide from the pancreas and continue to attempt simple cannulation for a maximum of 10 minutes or three UPCs. If three UPCs happen, we perform a TPP or, in certain specific cases with a higher risk of PAP, a NKP.

\section{Precutting technique}

Our pre-cutting technique has also been published previously [9] ( $\vee$ Video 2$)$. Briefly, we perform an initial superficial papillotomy (mucotomy) beginning at the papillary orifice and extending cranially approximately 5 to $15 \mathrm{~mm}$, depending on the size and condition of the papilla. After identifying the wall of the intraduodenal bile duct, we perform a second deep fistulotomy (myotomy) several cranial millimeters to the papillary orifice. Once the NKP is complete, we reattempt the BC using the $\mathrm{CT}$ and the hydrophilic guide. After a BC, we always perform a

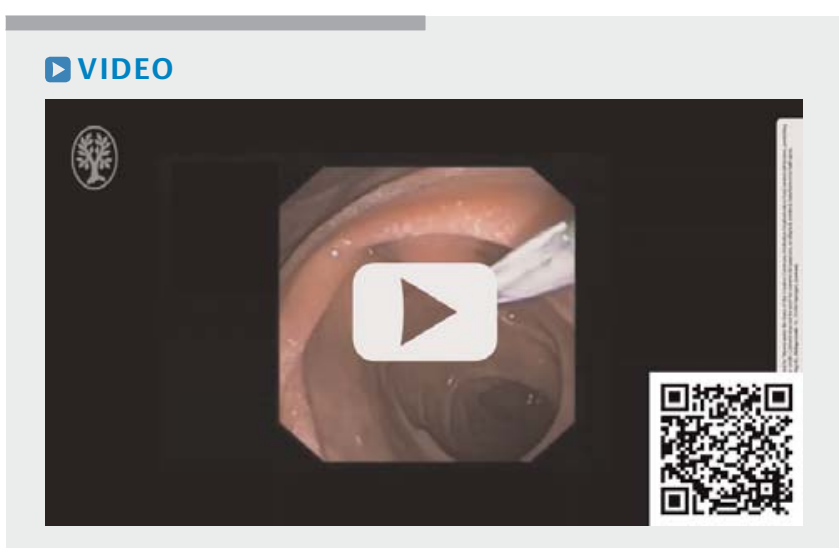

Video 2 In vivo performance of the OC-HT.

traction papillotomy of an appropriate size for the papillary anatomy and the therapeutic indications.

\section{Study population}

The study was conducted on all patients for whom BC was indicated and where the papilla was accessible and native. We excluded patients for whom pancreatic intervention was indicated or who had an inaccessible or previously treated papilla (by ampulectomy, papillotomy, or biliary or pancreatic stenting). The analysis included the consecutive procedures where a NKP 


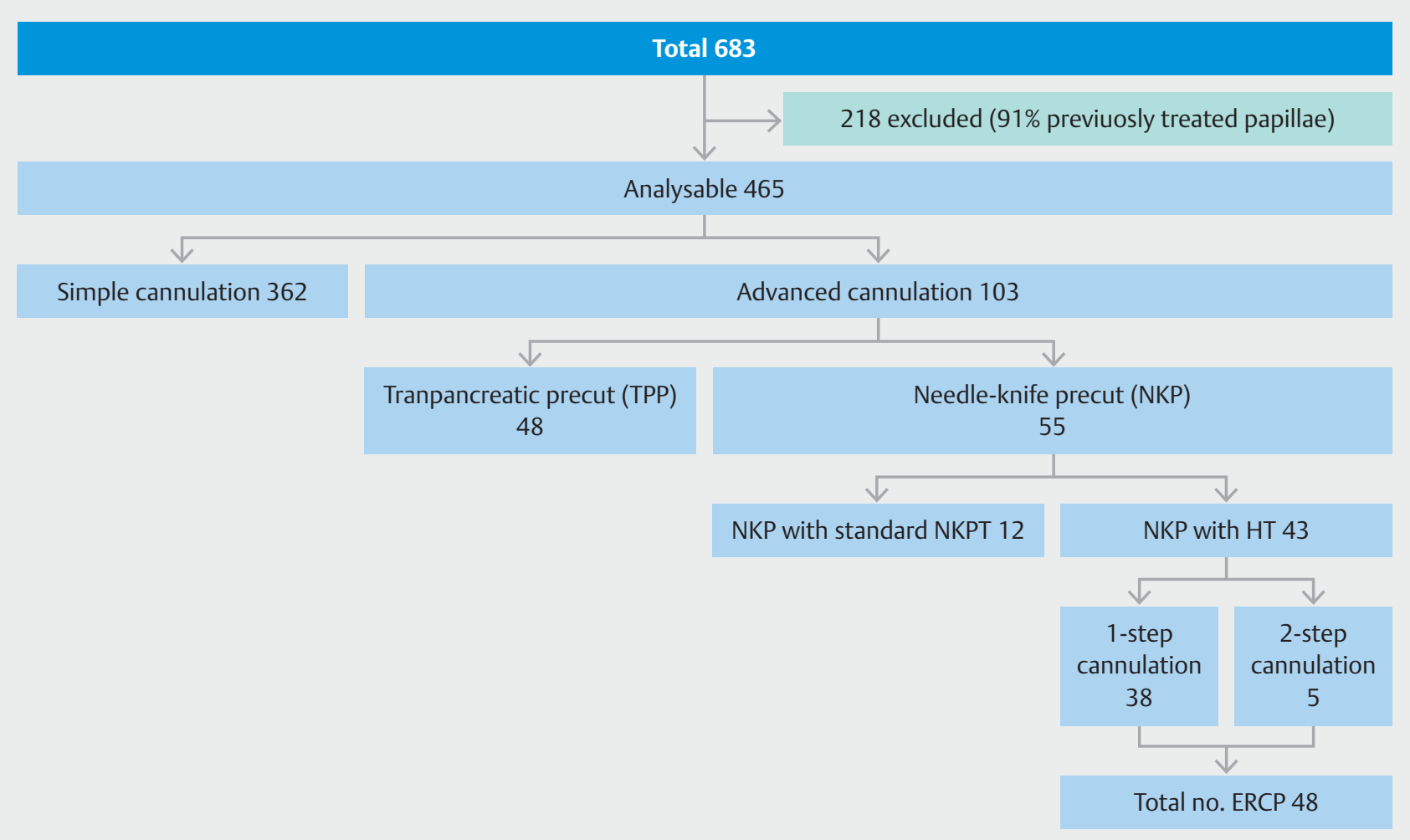

Fig. 3 Distribution of patients undergoing ERCP according to the biliary cannulation technique used during the study period.

was performed using a HT. In neither case did we try the NKP previously with a standard NKPT nor this was dismissed because of the papillary anatomy or alignment. We excluded those performed using the standard NKPT and combined precuts where TPP was performed first. Combined precuts in which the NKP was performed first were included. The introduction of the HT was not simultaneous in both hospitals but six months earlier in the M hospital. In those 6 months of delay, seven NKPs with the standard NKPT were performed in the $Z$ hospital. Procedures completed in two steps are counted as one (patient) for the efficacy analysis and as two (ERCP) for the safety analysis. All patients signed before the procedure a standard ERCP informed consent document. The retrospective analysis for the study was approved by the ethical committees in both hospitals.

\section{Analysis of results}

We defined technical success as achieving a $B C$ on the first or second attempt if the clinical situation did not require immediate biliary drainage during the first attempt. The adverse events (AEs) recorded were defined and graduated according to up-todate consensus criteria [10]. The incidence of AEs was compared to that recorded in our previous study [9], which included procedures performed in the period 2013-2016, for which reason there was an overlap between the second half of 2016 and the period of this study. We descriptively analyzed all the sociodemographic and clinicals variables, expressing quantitative variables through means and standard deviations and qualitative variables with percentages, using the statistical package
IBM SPSS Statistics 24, applying the test $x^{2}$ for independent data.

\section{Results}

Between May 2016 and April 2020, we performed 683 ERCP, of which 465 were able to be analyzed. Two hundred and eighteen procedures satisfied some of the exclusion criteria, $91 \%$ for having been performed on previously intervened papillae. In 362 ERCP, BC was obtained using the simple method and in 103 (22\%), an advanced cannulation method was necessary. In 48 cases a TPP was performed and in 55 a NKP, of which 12 were conducted with a standard NKPT and 43 with an HT. Apart from the seven NKPs with NKPT performed in the $Z$ hospital because of the delay of 6 months in the introduction of the HT, another five were performed because of the lack of a pre-assembled HT at the beginning of the ERCP. Thirty-eight of the $43 \mathrm{HT}$ procedures were completed in one step and five in two steps. As a result, our final study group comprised 43 patients who underwent 48 ERCPs ( $\mathbf{F i g . 3}$ ). Sixteen were treated at M hospital and 27 at $Z$ hospital.

Twenty-four (56\%) were women, with a mean age of $68.5 \pm$ 15 years (range 34-100). Nineteen had normal papillae, six were peri- or intradiverticular, 13 were inflammatory with varying degrees of severity, four had impacted stones, and one was neoplastic. The final diagnosis was choledocholithiasis in $18 \mathrm{pa}-$ tients, acute papillitis in four, stenosing papillitis (=SOD-I) in eight, SOD-II in one, ampullary carcinoma in one, pancreatic 
carcinoma in seven, and pancreatic neuroendocrine tumor in one. No active extravesicular pathology was found in three patients.

In 19 procedures we used the OC-HT, in 12 we used the CC$\mathrm{HT}$ and in 12 the BSC-HT. BC was obtained in all patients, a second time in five of them. In one patient who needed two steps, the first combined NKP and TPP. After BC, a traction papillotomy was performed in all cases. In addition to removing the stones found, eight biliary dilations were performed and nine biliary stents were implanted. In all the procedures, except for two patients admitted to the Intensive Care Unit (ICU), $100 \mathrm{mg}$ of rectal indomethacin was administered. Two patients at high risk of PAP received hyperhydration with Ringer's solution. No preventive pancreatic stents were implanted.

We recorded 11 AEs (23\%): five hemorrhages, four cases of pancreatitis ( 1 severe), and two cases of cholangitis. All occurred after the first step except for one bleed attributable to the traction papillotomy performed in the second step.

The $100 \%$ technical success was matched by a $95 \%$ clinical success rate (41 patients). Both patients admitted to the ICU died, one from biliary septic shock with multiorganic failure that was already established when the ERCP was performed; the other death was caused by cardiogenic shock initially attributed to a biliary etiology suggested by biliary dilatation detected in a CT scan, but which was later ruled out by the ERCP.

\section{Discussion}

Our first intervention on a human patient using a HT was performed in March 2016. It was a patient submitted to a previous emergency ERCP for biliary sepsis and in whom a biliary plastic stent was implanted, with no prior papillotomy, due to its anticoagulation status. Once this had been corrected, we performed the papillotomy in a second step. Our intention was to perform the first operation with the added security provided by the stent. This case was included in our preliminary communication [11], but excluded from this study because the patient had been cannulated previously and had a transpapillary stent when the NKP was performed.

Our idea is simple and not totally original, because it has been developed independently by at least one other author [12], but we are surprised that it has not been adopted and developed further by the medical industry. The traction capacity applied to the cannulation device makes the simple BC technique significantly easier and it has been universally implemented. Similarly, we consider this to be the logical progression from NKPT to facilitate its use, both by endoscopists in training as well as those who have already been trained.

Beyond the objective results, which we will discuss below, the subjective experience with the HT has been positive, and we found it easier to align with the ideal cutting plane and axes. Surprisingly, we discovered that to achieve this alignment, the need to bend the HT through the traction of the CT handle is small, because the natural curvature of the CT achieves this by itself in a high proportion of cases. This curvature proper of the $\mathrm{CT}$ is firm and sustained, unlike the pre-curvature with which some NKPT models are prepacked. Some- times, however, particularly in intradiverticular papillae and those hidden under fixed folds, there is a greater need for traction to perform the initial superficial papillotomy. Typically, the later deep fistulotomy requires no additional traction. This fact partially compensates for the major inconvenience we encountered, involving the effect that $C T$ traction causes by projecting the needle forward, and which requires compensatory retraction. Consequently, the HT makes NKP easier for the endoscopist, but complicates the role of the assistant who controls both handles, because they must manipulate them in a coordinated manner, compensating with one hand for the effect caused by the other, so that the net result on the needle is neutral.

This effect, observed equally with the three devices tested, could perhaps be attenuated by a device designed specifically for this purpose. However, we believe that the greatest functional benefit of our idea lies in the design of a needle that can be used in any type of $\mathrm{CT}$, including those that have no Luer connector at the mouth of the channel, and which can be quickly and easily interchanged with the cannulation guide. The ideal mechanics would be:

1. Attempting cannulation with $C T$ and guidance up to the point that the criteria for DBC are met.

2. Exchanging the guide for the needle-knife.

3. Performing NKP.

4. Exchanging the needle-knife for the guide.

5. Attempting cannulation once more.

Our working mechanics have been more complex since we performed the initial simple cannulation attempt with the CT Autotome RX-44 (Boston Scientific, Natick Massachusetts, United States), whose guide channel has a specific design for the rapidexchange function and to which the needle cannot be fixed by means of a Luer connection. Consequently, performing the NKP requires us to remove this device, introduce the previously assembled HT, remove this once the NKP has been performed, and reintroduce the initial $C T$ to complete the $B C$ and perform the traction papillotomy.

Aside from the extra cost of using one additional device (the second (T), HT assembly and testing during the ERCP means a longer procedure. For this reason, we chose to have the HT previously assembled and tested. However, with previous assembly, because when it will be used is unknown, requires a re-sterilization cycle, which represents an additional cost. Moreover, that was the reason that in five cases, we did not have the HT in time and fell back on standard NKP. For all these reasons, the design and performance of the homemade device is inefficient and must evolve before it can be summited to obtain Conformité Européenne marking and be available for clinical application.

With regard to the objective results achieved, we can confirm a technical success of $100 \%$ and a high rate of clinical success. The two fatalities recorded were not attributable to technical factors but to the delay in indicating ERCP and performing biliary drainage in one, and to the unsuitability of ERCP in the other, as there was no active biliary pathology. 
In terms of AEs, we recorded a certain change in pattern with respect to our previous study [9], in which there were none of 53 PAP, while in this study, the figure rose to four of 48 PAP (8.3\%) $(P=0.032)$. These data are even more surprising, considering that during the previous study, rectal indomethacin was administered progressively, while in this study, it was given systematically. Although we found no explanation for this phenomenon, we do not believe that it was caused by use of HT instead of NKPT, since the needle-knife, diathermy parameters, and the technique employed were the same. In contrast, in the previous study, there were three perforations in 53 procedures $(5.7 \%)$, while in this study, we recorded none $(P=0.094)$. Because the objective of HT is to facilitate technical performance of a NKP, we cannot rule out the possibility that the change of device influenced this fact, which we still consider beneficial even if this benefit is not statistically significant. We have no evidence to support this, except for the results of a classical study that reported NKP as being a risk factor for perforation and not for PAP [13].

\section{Conclusions}

The present results suggest that HT makes the technical performance of NKP easier for trained and experienced endoscopists, particularly when faced with intradiverticular or anatomically concealed papillae beneath duodenal folds. Such a hypothesis must be evaluated in comparative trials, but at this time, we consider it a priority to improve the design and standardize the handling to make the device's use more efficient.

\section{Acknowledgements}

The authors thank Cristina Antón Rodríguez, from the Investigation Support Unit, Medicine Faculty, Francisco de Vitoria University, for her critical revision and translation of the manuscript.

\section{Competing interests}

The authors declare that they have no conflict of interest.

\section{References}

[1] Testoni PA, Mariani A, Aabakken L et al. Papillary cannulation and sphincterotomy techniques at ERCP: European Society of Gastrointestinal Endoscopy (ESGE) clinical guideline. Endoscopy 2016; 48: 657-683

[2] Liao W, Angsuwatcharakon P, Isayama H et al. International consensus recommendations for difficult biliary access. Gastrointest Endosc 2017; 85: 295-304

[3] Kim S], Kang DH, Kim HW et al. Needle-knife fistulotomy vs doubleguidewire technique in patients with repetitive unintentional pancreatic cannulations. World J Gastroenterol 2015; 21: 5918-5925

[4] Gong B, Hao L, Bie L et al. Does precut technique improve selective bile duct cannulation or increase post-ERCP pancreatitis rate? A metaanalysis of randomized controlled trials Surg Endosc 2010; 24: $2670-$ 2680

[5] Choudhary A, Winn J, Siddique $S$ et al. Effect of precut sphincterotomy on post-endoscopic retrograde cholangiopancreatography pancreatitis: a systematic review and meta-analysis. World J Gastroenterol 2014; 20: 4093-4101

[6] Navaneethan U, Konjeti R, Venkatesh PGK et al. Early precut sphincterotomy and the risk of endoscopic retrograde cholangiopancreatography related complications: an updated meta-analysis. World J Gastrointest Endosc 2014; 6: 200-208

[7] Sundaralingam P, Masson P, Bourke M]. Early precut sphincterotomy does not increase risk during endoscopic retrograde cholangiopancreatography in patients with difficult biliary access: a meta-analysis of randomized controlled trials. Clin Gastroenterol Hepatol 2015; 13 : 1722-1729

[8] Baillie J. Reflections on needle-knife papillotomy. Gastrointest Endosc 2014; 79: 822-827

[9] Morena MadrigalEJ, Rodríguez GarcíaMI, Galera RódenasAB et al. Biliary cannulation effectiveness and pancreatitis risk using two early precut techniques. Rev Esp Enferm Dig 2018; 110: 74-81

[10] Cotton PB, Eisen GM, Aabakken L et al. A lexicon for endoscopic adverse events: report of an ASGE workshop. Gastrointest Endosc 2010; 71: 446-454

[11] Morena-Madrigal EJ. A “Hybrid-tome” for biliary needle-knife precut: Preliminary results. Gastrointest Endosc 2018; 870: AB201

[12] Keskin M. A novel combined needle-knife and standard sphincterotome: the dualtome. Endoscopy 2016; 48: E396-E397

[13] Loperfido S, Angelini G, Benedetti G et al. Major early complications from diagnostic and therapeutic ERCP: a prospective multicenter study. Gastrointest Endosc 1998; 48: 1-10 